\title{
Ventajas de la tecnología Near Field Communication (NFC) como sistema de pago electrónico
}

\author{
Advantages of Near Field Communication (NFC) technology as electronic payment \\ system \\ Vantagens da tecnologia Near Field Communication (NFC) e sistema de \\ pagamento eletrônico
}

Rogelio Marcelino Avilés

Centro Universitario Temascaltepec, Universidad Autónoma del Estado de México, México

ro.j.08@hotmail.com

\section{Resumen}

El presente trabajo se realizó con el objetivo de estudiar las ventajas del uso de la tecnología Near Field Communication" o "Comunicación de campo cercano", como sistema de pago electrónico, mediante el uso de un móvil con capacidad de almacenar aplicaciones de pago que son compatibles con los millones de puntos de venta sin contactos existentes actualmente, realizando un análisis documental donde se demuestre que son más las ventajas de usar un teléfono móvil para realizar los pagos, que los métodos tradicionales.

Para el desarrollo del presente estudio se investigaron y analizaron las diversas fuentes de información relacionadas con el tema en el año 2014.

Se llegó a la conclusión de que es más cómodo y seguro pagar con dispositivos con tecnología NFC que con un sistema tradicional, ya que ofrece mayor seguridad que los sistemas que actualmente se usan como herramienta para pagar electrónicamente.

Palabras clave: NFC, pago móvil, pago electrónico. 


\section{Abstract}

The present work was carried out with the aim of studying the advantages of the use of Near Field Communication Technology as electronic payment system, through the use of a mobile phone with the ability to store payment applications that are currently compatible with the millions of points of sale without existing contacts, making a documentary analysis which proves that they are more the advantages of using a mobile phone to make payments than traditional methods.

The development of the present study is investigated and analysed the various sources of information related to the subject in the year 2014 .

It was concluded that it is more comfortable and safe to pay with devices with NFC than with a traditional system, since it offers greater security than systems currently used as a tool to pay electronically.

Key Words: NFC, mobile payment, electronic payment.

\section{Resumo}

Este trabalho foi realizado com o objetivo de estudar as vantagens do uso da tecnologia Near Field Communication "ou" Near Field Communication ", como sistema de pagamento eletrônico, usando um capaz móvel de armazenamento de aplicativos de pagamento são compatível com os milhões de pontos de venda Atualmente não há contatos existentes, fazendo uma análise documental, onde se for provado que há mais vantagens do uso de um telefone celular para fazer pagamentos, que os métodos tradicionais.

Para o desenvolvimento deste estudo foram investigados e analisados os diferentes fontes de informação relacionada com o tema em 2014.

Concluiu-se que é mais confortável e seguro para pagar com dispositivos NFC do que com um sistema tradicional, proporcionando uma maior segurança do que os sistemas actualmente utilizados como uma ferramenta para pagar electronicamente.

Palavras-chave: NFC, pagamento móvel, pagamento eletrônico.

Fecha recepción: Enero 2016

Fecha aceptación: Junio 2016 


\section{Introducción}

El teléfono móvil desde hace unos cuantos años se ha convertido en un dispositivo indispensable dentro del estilo de vida digital en el que estamos inmersos. De ser un dispositivo que sólo servía para comunicarse con voz, se ha transformado en un dispositivo universal que incluye la funcionalidad de numerosos dispositivos en uno solo, como son cámara fotográfica, reproductor de música MP3, reproductor de video, navegador de internet, etcétera (Chavarría, 2014).

Como avance tecnológico de la comunicación, la creación e interconexión de redes de datos sólidas tiene un profundo efecto, ya sea en la comunicación entre dispositivos móviles portátiles que se utilizan actualmente, o en celulares, computadores, entre otros, y las nuevas tendencias tecnológicas a implementarse en ellos, que crece exponencialmente con el paso de los años. La tecnología inalámbrica NFC es un progreso en la convergencia de aplicaciones dentro del teléfono móvil, ya que ofrece los servicios de las tarjetas inteligentes y también las ventajas de las tecnologías inalámbricas de corto alcance. NFC presenta una característica particular y es su compatibilidad con las demás tecnologías inalámbricas ya existentes, como Bluetooth y RFID, lo que incrementa su inversión y desarrollo.

Recientes proyectos pilotos en Europa y Estados Unidos han puesto de manifiesto que la combinación de estas dos tecnologías puede permitir el desarrollo de más y nuevos servicios con un nivel de seguridad muy elevado, como el requerido para el pago a través del móvil. Son los denominados servicios de proximidad a los que el usuario puede acceder con tan sólo acercar su teléfono móvil a una terminal que ofrezca el servicio con más ventajas y la misma seguridad que las herramientas de pago que en la actualidad se utilizan para las distintas operaciones bancarias y/o pago de servicios. Una de las ventajas mayores a considerar es que diremos adiós a todas las tarjetas ya que el móvil se convertirá en nuestro monedero al ser sencillo de utilizar con un simple toque. 


\section{Objetivo general}

Analizar las ventajas de la tecnología NFC como sistema de pago electrónico.

\section{Metodología}

Para el presente trabajo se utilizó la metodología documental. Según Baena (1985) es una técnica que consiste en la selección y recopilación de información por medio de la lectura y crítica de documentos y materiales bibliográficos, de bibliotecas, hemerotecas, centros de documentación e información (Baray, 2006).

\section{Materiales y métodos}

La presente investigación se basó en el análisis de artículos científicos, documentos, foros, ensayos, casos de éxito, etcétera.

\section{NFC (Near Field Communication o Comunicación de campo cercano)}

NFC es un sistema de transmisión de datos similar al bluetooth, que utiliza los principios de la tecnología RFID (identificación por radiofrecuencia). Sin embargo, ofrece prestaciones mucho más amplias que la RFID porque aprovecha el extendido uso de los teléfonos móviles y sus capacidades de cómputo (nfc-forum, 2014).

La comunicación en campo cercano, usualmente llamada NFC, es una tecnología de comunicación inalámbrica a corto alcance y elevada frecuencia de $3.56 \mathrm{MHz}$, lo que permite el intercambio de información entre periféricos hasta una distancia de aproximadamente $10 \mathrm{~cm}$. Esta tecnología es una extensión de la norma ISO/IEC 144437 que estandariza los mapas de proximidad que utiliza la RFID, combinando una interfaz de un mapa a chip y un lector dentro de un sólo periférico mediante un estándar ISO, ECMA y ETSI que trabaja en la banda de frecuencia AF (frecuencia alternativa) (13,56 MHz).

Actualmente ofrece velocidades de transmisión de datos de 106 kbps, 212 kbps y 424 kbps y no está pensado para transmitir grandes volúmenes de datos, sino más bien para intercambiar información de forma rápida, eficiente y segura. Al igual que el resto de tecnología RFID, el protocolo NFC cubre los modos de operación activo y pasivo (Reyes, 2014). 
La tecnología NFC es concebida para su uso en los teléfonos móviles; se pedirá al usuario escanear su huella dactilar o ingresar un código secreto para aprobar la transacción, validando la transacción con un chip por separado que se conoce como SE (elemento de seguridad) que comunica la autorización al módem NFC. De ahí, el pago se termina de procesar de la misma manera como se finaliza una transacción en la que se desliza una tarjeta de crédito o débito en una caja registradora (Profis, 2014).

Su desarrollo empieza en el año 2002 y sus promotores fueron Philips y Sony principalmente para conseguir compatibilidad con sus tecnologías Mifare y FeliCa, pero fue a finales del año 2003 que se aprobó como el estándar ISO 18092 (Sacristana, 2014).

Con el fin de lograr la mayor penetración de NFC, Sony y Philips decidieron en 2002, a través de ECMA internacional, crear el estándar abierto 340 "NFC interface and protocol", el cual fue adoptado en el 2003 por ISO/IEC con el número 18092. Para lograr una mayor promoción de NFC, Nokia, Philips y Sony fundaron en 2004 el NFC fórum.

\section{Sistemas de pago electrónico en la actualidad}

\section{Tarjeta electrónica}

La tarjeta como medio de pago engloba un conjunto muy amplio de distintos productos (tarjetas de crédito, de débito o monedero). Sin embargo, todas tienen dos elementos comunes: la vinculación del cliente, comercio o ambos a una cuenta bancaria necesaria para poder finalizar las transacciones, y la dificultad y lentitud en el proceso de aceptación de este medio de pago.

La tarjeta electrónica presenta modificaciones respecto al esquema tradicional. El proveedor transmite la información a su banco y éste al sistema (Santoma, 2014).

\section{Cajeros electrónicos}

Los cajeros automáticos también conocidos como ATM (Automatic Teller Machine), son dispositivos cuya finalidad principal es dispensar efectivo, además de realizar las siguientes 
operaciones financieras: consultar saldos y movimientos, compra de tiempo aire para teléfonos celulares, pago de servicios, cambio de NIP (clave confidencial), etcétera (Bancomer, 2013).

\section{Cheques electrónicos}

Son transacciones electrónicas de una vez que hace un minorista contra tu cuenta para el cheque que le acabas de escribir. En vez de procesar el cheque de papel que le diste, el minorista toma el nombre, el número de ruta, el número de cheque y otra información pertinente del cheque y proceso una transferencia de fondos única de tu banco al suyo. Te devuelve el cheque junto con tu recibo (Finazas, 2013).

Un cheque es un documento utilizado como medio de pago por el cual una persona (el librador) ordena a una entidad bancaria (el librado) que pague una determinada cantidad de dinero a otra persona o empresa (el beneficiario o tenedor). El librador puede ser también el beneficiario, como ocurre cuando uno utiliza un cheque para sacar dinero de su propia cuenta (Plan de Educacion, 2010).

\section{Dinero electrónico anónimo fuera de línea}

Con el dinero electrónico anónimo fuera de línea (off-line) el comerciante no tiene que interactuar con el banco antes de aceptar dinero por parte del usuario. En lugar de eso puede recoger múltiples monedas gastadas por los usuarios y depositarlas posteriormente en el banco. En principio esto se puede hacer fuera de línea, es decir, el comerciante podría ir al banco con sus medios de almacenamiento para intercambiar el efectivo electrónico por dinero en efectivo. Asimismo, el comerciante debe asegurarse de que el dinero electrónico del usuario, o bien sea aceptado por el banco, o que el banco sea capaz de identificar y castigar a los usuarios que traten de engañar por esta vía. De esta forma, un usuario no tiene posibilidad de utilizar la misma moneda dos veces. Los sistemas de efectivo electrónico off-line también tienen la necesidad de protegerse contra los posibles engaños de los comerciantes, es decir, los comerciantes que deseen depositar una moneda dos veces (Canal asesor, 2013). 


\section{Ventajas y desventajas del uso de la tecnología NFC Ventajas del uso de la tecnología NFC}

El uso de la tecnología NFC tiene grandes ventajas, las cuales le permiten al usuario liberarse de cables para compartir información entre dispositivos móviles, recargarlos o lograr con un toque que smartphones, tabletas, equipos de audio, sistemas de pago, vehículos y electrodomésticos ‘hablen' e interactúen. Este es uno de los adelantos alcanzados por la tecnología inalámbrica de corto alcance (El tiempo, 2014).

Como si fuese un truco de magia, en el que las conexiones físicas desaparecen para transmitir información, los equipos y plataformas que trabajan con NFC permiten, en segundos, conectar un dispositivo con múltiples aparatos móviles a través de la identificación de radiofrecuencia (RFID).

La tecnología, que se prueba desde el 2004, despegó en Europa en el 2010 con los primeros ensayos para realizar transacciones financieras a través de teléfonos móviles.

A raíz de esto, compañías como Visa, Master Card, Telefónica, Citibank y Google, con su Google Wallet, entre otros, ya cuentan con sistemas de pago con NFC desde el 2011, convirtiendo así a los smartphones en billeteras inteligentes que ofrecen muchas ventajas (El tiempo, 2014).

- La más obvia de todas es la comodidad, diremos adiós a todas las tarjetas ya que el móvil se convertirá en nuestro monedero.

- Es sencillo de utilizar, se hace a través de un simple toque.

- Ofrece una mayor personalización, recopila datos de los clientes de manera que permite conocerlos mejor.

- Ahorro de tiempo, tanto en pagos en tiendas, bares y restaurantes, como en medios de transporte. Resulta mucho más cómodo subir al autobús y acercar el móvil al dispositivo que tener que adquirir el billete físico.

- Va más allá del pago, pues la tecnología NFC ofrece otros usos. Desde la posibilidad de canjear cupones de descuento hasta realizar campañas de social media a través de los 
dispositivos móviles, por ejemplo, marcar un check in en Foursquare, comenzar a seguir en Twitter o hacerte fan en Facebook de un local simplemente acercando tu móvil a los logos del establecimiento.

- La posibilidad de asociar un número de cuenta bancaria a nuestro dispositivo móvil permite ganar en comodidad y rapidez a la hora de validar compras con la única acción de acercar el teléfono a un equipo receptor.

- Facilidad para entrar en puntos donde sea necesario una identificación.

- El tiempo de conexión entre los dos dispositivos es extremadamente rápido, ya que tan solo lleva 0.1 segundos y se hace automáticamente.

- Tiene muchos más usos que bluetooth, entre los que destacan, por ejemplo, el pago de servicios con el celular mediante NFC con la aplicación de Google Wallet.

\section{Desventajas del uso de la tecnología NFC}

- La velocidad es más lenta que la de bluetooth, ya que es de $424 \mathrm{kbits} / \mathrm{s}$ mientras que la de bluetooth es de $2.1 \mathrm{Mbit} / \mathrm{s}$.

- La distancia máxima a la que se pueden tener los dos dispositivos o aparatos tecnológicos es de $20 \mathrm{~cm}$. Esto hace que los teléfonos móviles deban estar juntos. Tiene otro uso en comparación con bluetooth.

- Únicamente la conexión entre los artefactos es de modo punto a punto, no hay posibilidad de crear una red inalámbrica entre varios dispositivos.

- Se trata de una comunicación por radiofrecuencia, por lo que siempre existe la posibilidad de que se realice una lectura de la transmisión.

- Se opera a poca distancia, por lo que se pueden copiar los códigos para uso fraudulento (Gigactecno, 2014).

\section{Seguridad NFC}

Uno de los aspectos que más desconfianza despierta es la seguridad, aunque lo cierto es que es tan seguro como una tarjeta de crédito. Las transacciones se aseguran con los mismos métodos que las tarjetas; el chip NFC no tiene acceso a los datos del móvil, así que no se puede acceder a él a través de programas maliciosos. En caso de robo del móvil, el ladrón necesitaría la 
contraseña para acceder al dinero; además el chip NFC se puede bloquear del mismo modo que se hace con las tarjetas de crédito (Seguridad del uso de la tecnologia NFC, 2013).

Ya sea un chip en el teléfono, o desde la nube, el elemento de seguridad es a prueba de manipulaciones y está protegido por una firma digital única. De acuerdo con Michael Armentrout, fabricante de chips de elementos de seguridad Infineon, la arquitectura del elemento de seguridad está diseñada contra ataques al teléfono (Profis, 2014).

\section{Pago móvil}

Es la forma de pago que te permite realizar compras por internet y por teléfono con tu celular y sin proporcionar los datos reales de tus tarjetas, ya que se genera una tarjeta virtual distinta y personalizada en cada transacción, ofreciendo máxima seguridad en compras electrónicas (Giesecke y Devient, 2014).

Los teléfonos con capacidad NFC pueden almacenar aplicaciones de pago compatibles con los millones de puntos de venta sin contactos existentes actualmente.

La intuitiva simplicidad de acercar un teléfono móvil a un Terminal Punto de Venta (TPV) para pagar por productos o servicios, contrasta con la dificultad de leer o insertar una tarjeta en una ranura de un TPV (Giesecke y Devient, 2014).

Además, un teléfono puede almacenar información sobre múltiples cuentas como puede ser de crédito, débito o prepago, permitiendo al usuario seleccionar el método de pago más adecuado en cada situación de forma mucho más sencilla que tener que llevar múltiples tarjetas en el monedero.

Las transacciones son totalmente seguras y la aplicación de pago está protegida mediante contraseña. En caso de pérdida o robo del teléfono es posible desactivar la aplicación a distancia aumentando aún más la seguridad. 


\section{Discusiones}

Hoy en día existe un amplio número de sistemas electrónicos intercambiarios de fondos bancarios que aseguran la circulación de dinero. Nuestro estudio encontró que las ventajas de la tecnología NFC como medio de pago electrónico rompe paradigmas. Antes no se vislumbraba que las personas pudieran decir adiós al efectivo, las tarjetas de débito o crédito, pero ahora es una realidad.

\section{Conclusiones}

NFC aparece como un progreso en la convergencia de aplicaciones dentro del teléfono móvil al ofrecer los servicios de las tarjetas inteligentes: crédito y débito. Gracias a esta nueva tendencia tecnológica el usuario contará con la misma comodidad y seguridad que los sistemas de pago tradicionales.

Cabe mencionar que esta tecnología tendrá un impacto positivo en la sociedad, ya que gracias a su uso las personas dirán adiós a todas las tarjetas electrónicas. El teléfono se convertirá en su monedero personal, y mediante una aplicación podrán realizar pagos de manera fácil y rápida. 


\section{Bibliografía}

Bancomer, B. (12 de enero de 2013). Servicios digitales. Recuperado el 13 de noviembre de 2014, de Cajeros Automáticos: https://www.bancomer.com/personas/cajerosautomaticos.jsp?nivel1 $=$ privada

Baray, H. L. (12 de marzo de 2006). Introducción a la metodología de la investigación. México: eumed.net. Obtenido de http://www.eumed.net/libros-gratis/2006c/203/2c.htm

Canal asesor (06 de febrero de 2013). El dinero electrónico. Recuperado el 19 de octubre de 2014, de http://canalasesor.wke.es/ver_detalleArt.asp?idArt=67147\&action=ver

Chavarría, D. A. (24 de febrero de 2014). ucr. Obtenido de http://eie.ucr.ac.cr/uploads/file/proybach/pb2011/pb2011_012.pdf

El tiempo (octubre de 27 de 2014). Aprenda las ventajas de usar la tecnología NFC. Recuperado el 21 de noviembre de 2014, de http://www.eltiempo.com/archivo/documento/CMS12590221

Finazas (23 de febrero de 2013). Cheques electrónicos. Recuperado el 19 de octubre de 2014, de http://www.ehowenespanol.com/cheque-electronico-funciona-sobre_438560/

Giesecke y Devient . (23 de febrero de 2014). NFC: Transacciones seguras. Recuperado el 19 de noviembre de 2014, de http://www.gide.com/es/trends_and_insights/nfc_mobile_phones/nfc-mobile-phones.jsp

Gigactecno (1 de septiembre de 2014). Gigactecno. Obtenido de http://gigatecno.blogspot.mx/2014/09/ventajas-y-desventajas-de-nfc.html

nfc-forum (1 de enero de 2014). nfc-forum. Recuperado el 3 de junio de 2014, de nfc-forum: http://nfc-forum.org/

Plan de Educación (23 de febrero de 2010). Finanzas para todos. CNMV y Banco de España. Obtenido de Finanzas para todos. CNMV y Banco de España: http://www.finanzasparatodos.es/es/productosyservicios/productosbancariosoperativos/m ediosdepagocheques.html

Profis, S. (2014). CNET. Recuperado el 8 de septiembre de 2014, de https://www.cnet.com/es/noticias/lo-que-debes-saber-de-nfc-y-los-pagos-moviles/

Reyes, C. A. (14 de marzo de 2014). cybertesis. Obtenido de http://cybertesis.uach.cl/tesis/uach/2014/bmfcia775a/doc/bmfcia775a.pdf

Sacristana, F. G. (12 de enero de 2014). uc3m. Obtenido de uc3m: http://earchivo.uc3m.es/bitstream/handle/10016/13738/PFC_Fermin_GallgoSacristana_LopezPa blo.pdf?sequence $=1$

Santoma, J. (17 de julio de 2014). IESE. Obtenido de http://www.iese.edu/research/pdfs/ESTUDIO-18.pdf 
Seguridad del uso de la tecnología NFC (21 de septiembre de 2013). Tecnología NFC. Recuperado el 29 de octubre de 2014, de http://ldc.usb.ve/ figueira/cursos/Seguridad/Expo/Presentaciones/SeguridadNFC.pdf 